\title{
Human Papillomavirus and Factors Associated with Recurrence in Sinonasal Inverted Papillomas from Poland and Spain
}

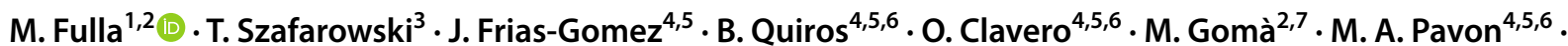 \\ O. Jurek-Matusiak ${ }^{3} \cdot$ H. R. Lares ${ }^{1,2} \cdot$ M. Mañós $s^{1,2,8} \cdot$ L. Alemany ${ }^{4,5,9} \cdot$ M. Mena ${ }^{4,5,6} \cdot$ X. Gonzalez ${ }^{1,2}$
}

Received: 7 October 2019 / Accepted: 30 December 2019 / Published online: 8 January 2020

๑) Springer Science+Business Media, LLC, part of Springer Nature 2020, corrected publication 2020

\begin{abstract}
Sinonasal inverted papilloma (SNIP) is a benign but locally aggressive tumor that has a tendency for recurrence and malignant transformation. The role of human papillomavirus (HPV) in SNIP is controversial. To determine the HPV-DNA prevalence and type distribution in SNIP in two different geographic areas and assess the association between SNIP recurrence and HPV infection, as well as additional potential etiologic factors. Two retrospective cohorts of SNIP patients from Poland and Spain were evaluated. Demographic, tobacco/alcohol use, clinical, and follow-up data were collected. All samples were subject to histopathologic evaluation, DNA quality control, and HPV-DNA detection by PCR. HPV-DNA positive samples and a random sample of HPV-DNA negative cases were further subject to $\mathrm{p} 16^{\mathrm{INK} 4 \mathrm{a}}$ analysis. Proportional-hazards models were used to evaluate the risk of recurrence by selected variables. Seventy-nine SNIP patients (46 from Spain diagnosed between 1995 and 2014, and 33 from Poland diagnosed between 2012 and 2017) were included in the study. HPV-DNA was detected in four patients (5.1\%), two from each region, and all four were positive for the HPV11 subtype. Seventeen patients (21.5\%) experienced recurrence, with a median time to recurrence of 14 months. No association was identified between lesional HPV-DNA positivity, toxic habits, Krouse stage, or malignant transformation and a higher risk of recurrence. The low prevalence of HPV-DNA in SNIPs suggests that HPV is not a main etiology for development of these lesions. With a lack of association between the evaluated factors and recurrence, further research with larger number of patients and additional biomarkers is warranted to further understand predisposing risk factors.
\end{abstract}

Keywords Inverted papilloma $\cdot$ Schneiderian papilloma $\cdot$ Sinonasal papilloma $\cdot$ Human papillomavirus $\cdot$ HPV

M. Mena and X. Gonzalez are co-senior authors.

Electronic supplementary material The online version of this article (https://doi.org/10.1007/s12105-019-01125-y) contains supplementary material, which is available to authorized users.

M. Fulla

mfulla@bellvitgehospital.cat

1 Department of Otorhinolaryngology, Hospital Universitari Bellvitge, L'Hospitalet de Llobregat, Barcelona, Spain

2 Program of Molecular Mechanisms and Experimental Therapy in Oncology, Bellvitge Biomedical Research Institute (IDIBELL), L'Hospitalet de Llobregat, Barcelona, Spain

3 Department of Otorhinolaryngology, Faculty of Medicine and Dentistry, Medical University of Warsaw, Warsaw, Poland

4 Cancer Epidemiology Research Program, Catalan Institute of Oncology (ICO) - L'Hospitalet de Llobregat, Barcelona, Spain
5 Epidemiology, Public Health, Cancer Prevention and Palliative Care Program, IDIBELL, L'Hospitalet de Llobregat, Barcelona, Spain

6 Centro de Investigación Biomédica en Red de Cáncer (CIBERONC), Instituto de Salud Carlos III, Madrid, Spain

7 Department of Pathology, Hospital Universitari Bellvitge, L'Hospitalet de Llobregat, Barcelona, Spain

8 University of Barcelona, Barcelona, Spain

9 Centro de Investigación Biomédica en Red de Epidemiología y Salud Pública (CIBERESP), Instituto de Salud Carlos III, Madrid, Spain 


\section{Introduction}

The sinonasal inverted papilloma (SNIP) is a benign epithelial neoplasm that represents $0.5-4 \%$ of primary sinonasal tumors and has an incidence of $0.2-0.7 / 100.000$ people/year. Occupational exposures such as organic solvents and welding fumes are implicated in the development of SNIPs [1]. Chronic sinonasal inflammation may also be a contributing factor, but the mechanism is unclear.

Sinonasal papillomas are classified according to their histologic structure as exophytic (fungiform) papilloma, inverted papilloma, and cylindrical or oncocytic papilloma, although all may coexist [1]. SNIP is the most common type and represents $70 \%$ of all sinonasal papillomas [2].

Within the sinonasal tract, SNIPs are most frequently found on the lateral wall of the nasal fossae, but may also occur in the ethmoidal and maxillary sinuses. Histologically, lesions show hyperplastic epithelial invaginations, ranging from squamous to ciliated columnar with goblet cells, into the underlying stroma (Figs. 1 and 2).

Although most are histologically benign, SNIPs are characterized by locally aggressive behaviour, and malignant transformation is reported in a subset [3]. Approximately $10 \%$ of SNIPs are associated with malignancy, most frequently squamous cell carcinoma (SCC) [4, 5]. The factors responsible for malignant transformation are not yet fully elucidated due to the relatively low prevalence of SNIPs and SCC arising in the sinonasal tract [4]. SNIPs are also prone to recurrence, although the postulated factors associated with recurrence, such as human papillomavirus (HPV) infection, tobacco smoking, or Krouse stage, have not been consistently corroborated [1, 6-8]. The recommended treatment is complete surgical excision by an endoscopic, open or combined, approach [9].

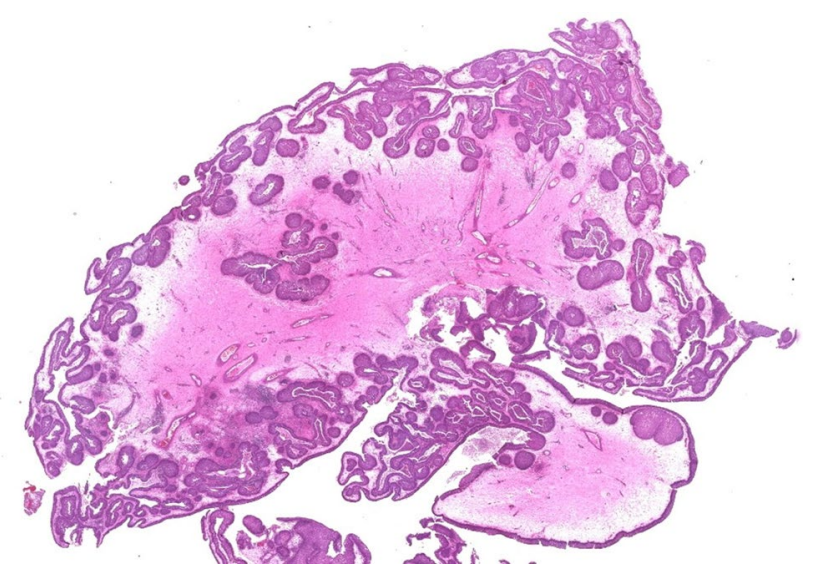

Fig. 1 Sinonasal inverted papilloma. Inverted growth pattern and absence of seromucinous glands (hematoxylin-eosin 0 '5x)

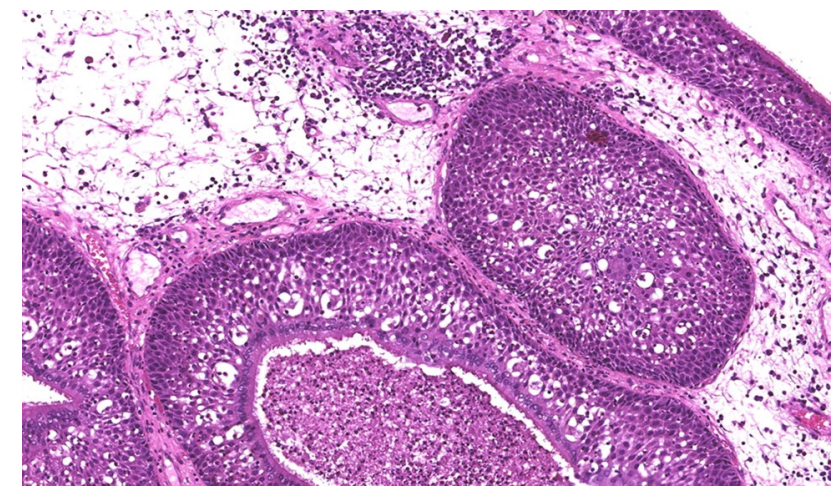

Fig. 2 High power image shows non-keratinizing transitional epithelium covered by a layer of ciliated columnar epithelium. Infiltration by neutrophils is seen (hematoxylin-eosin 10x)

The established role of HPV in a fraction of head and neck cancers (HNC) raised interest in the virus' etiologic and prognostic role in other benign head and neck lesions such as SNIP. In the last decades, several studies have explored the relationship between SNIP and HPV infection with conflicting results. The detection of HPV in SNIP ranges from 0 to $100 \%$ of lesions in the published literature $[1,3,10]$. Such differences have not been explained by varying geographic regions or HPV detection methods [10].

Recently, new theories postulate that HPV is not related to the initial pathogenesis of SNIPs, but the inflammatory and metaplastic mucosa of the lesion predispose it to viral infection [11]. Moreover, the presence of the virus in SNIPs has been related to a higher risk of recurrence and malignant transformation, especially for high-risk genotypes 16 and 18 $[3,6,12]$. The unequivocal establishment of a prognostic HPV role in SNIPs, as well as other sinonasal lesions, may have implications for tertiary prevention of recurrence or malignant transformation through vaccination.

This study aimed to estimate the HPV-DNA prevalence and type distribution in SNIPs in two series from different geographic areas, Spain and Poland, and to analyse risk factors for recurrence and malignant transformation in both groups.

\section{Methods}

\section{Study Design}

We carried out a retrospective study including two cohorts of all primary SNIPs diagnosed between 1995 and 2014 at the Department of Otorhinolaryngology of the Hospital Universitari de Bellvitge (Spain) and between 2012 and 2017 at the Department of Otorhinolaryngology of the Czerniakowski Hospital in Warsaw, Poland. The pathologic diagnosis of 
the lesions was confirmed by biopsy. Demographic data and information regarding smoking and alcohol use, previous HPV-related pathology, tumor extent by the Krouse staging system, and follow-up were collected from medical records [13]. Recurrent cases or those that previously underwent nasal surgeries were excluded.

Protocols were approved by the ethics committee of the Catalan Institute of Oncology-ICO (Comité Ètic d'Investigació Clínica de l'Hospital Universitari de Bellvitge, Spain) which required no informed consent to use archived samples.

\section{Formalin-Fixed, Paraffin-Embedded (FFPE) Block Processing and Histopathologic Evaluation}

Formalin-fixed, paraffin-embedded (FFPE) block processing protocols are described elsewhere [14]. Briefly, FFPE blocks were processed under strict conditions to avoid contamination and were re-embedded at ICO whenever necessary. At least four paraffin sections were obtained from each block. First and last sections were used for histopathologic evaluation (sandwich method) after hematoxylin and eosin (H\&E) staining. The in-between sections were used for HPV testing and genotyping as well as the expression of $\mathrm{p} 16^{\mathrm{INK} 4 \mathrm{a}}$. FFPE blocks were processed under strict pre/ post polymerase chain reaction (PCR) physical separation, and blank paraffin blocks were systematically tested in parallel to serve as sentinels for contamination as previously published [14]. Pathology review was blinded to the original local diagnosis and performed using a form specifically designed by two pathologists for the study (see supplementary material). It followed a pre-established algorithm for diagnostic consensus involving the two pathologists. First, all pathology slides were reviewed by a trained pathologist at ICO. Samples with a discordant diagnosis were further reviewed by the two pathologists for a final evaluation and agreed upon diagnosis.

\section{HPV-DNA Detection and Genotyping}

The detailed methods used for HPV-DNA detection and genotyping have been reported elsewhere [14]. Briefly, SPF-10 PCR and a DNA enzyme immunoassay (DEIA) was used to test for the presence of HPV-DNA. Virus genotyping was performed using a reverse hybridization line probe assay (LiPA25_v1) on all samples testing positive for viral DNA, targeting $25 \mathrm{HPV}$ types with oncogenic potential. DNA quality was evaluated in all HPV-DNA negative samples by testing for the human tubulin gene [14]. All DEIA and LiPA25_v1 assays were performed at ICO.

\section{p16 ${ }^{\text {INK4a }}$ Immunohistochemistry}

p16 ${ }^{\text {INK4a }}$ expression was evaluated on all HPV-DNA positive cases and a random sample of HPV-DNA negative cases using the CINtec histology kit (clone E6H4, Roche mtm laboratories AG, Germany) following the manufacturer's protocol. While a pattern of diffuse staining of more than $70 \%$ stained cells (nuclear and cytoplasmic) is considered positive for malignant lesions, staining between 26 and 50\% of cells for premalignant lesions, in a diffuse or continuous pattern, was considered positive in this study [15-17].

\section{Statistical Analysis}

Descriptive statistics were computed for each of the variables analysed. A Fisher's exact test for categorical variables and t-test for continuous variables were used to detect statistically significant differences between the two centres for each variable. Median and range of months to recurrence and months of follow-up variables were estimated. To compare medians between groups, the quantile regression (qreg) test, equivalent to t-test for means, was employed. A survival analysis was conducted to identify variables associated with recurrence, and a Cox regression model was performed to estimate hazard ratios and their $95 \%$ confidence interval (CI). In order to avoid possible bias due to the centre where the SNIP was diagnosed and treated, centre was introduced as a strata variable allowing the baseline hazard function to differ for each centre. Proportional hazard assumption was also verified. Due to the low number of cases progressing to invasive cancer during follow-up, survival analyses to identify variables associated with malignant transformation could not be performed. All the analyses were performed with STATA 13.1 software.

\section{Results}

Figure 3 summarizes the SNIP samples collected, processed, and tested. The ICO laboratory received 63 samples from Bellvitge Hospital (Spain) and 68 samples from Czerniakowski Hospital (Poland). A total of 79 cases (46 from Spain and 33 from Poland) were included in the final analysis.

The characteristics of the patients are presented in Table 1. Most were males (67.1\%) and non-drinkers (70.9\%) with a mean age of 56.2 years. There was a higher proportion of ever-smokers in the Spanish group $(67.4 \%$ vs $39.4 \%$, $\mathrm{p}=0.010$ ) whereas Polish cases were more frequently diagnosed with advanced Krouse stages $(p<0.001)$. Differences in histopathologic features were observed between the two centres with more Polish cases displaying transitional 
Fig. 3 Flow chart of cases included in the study

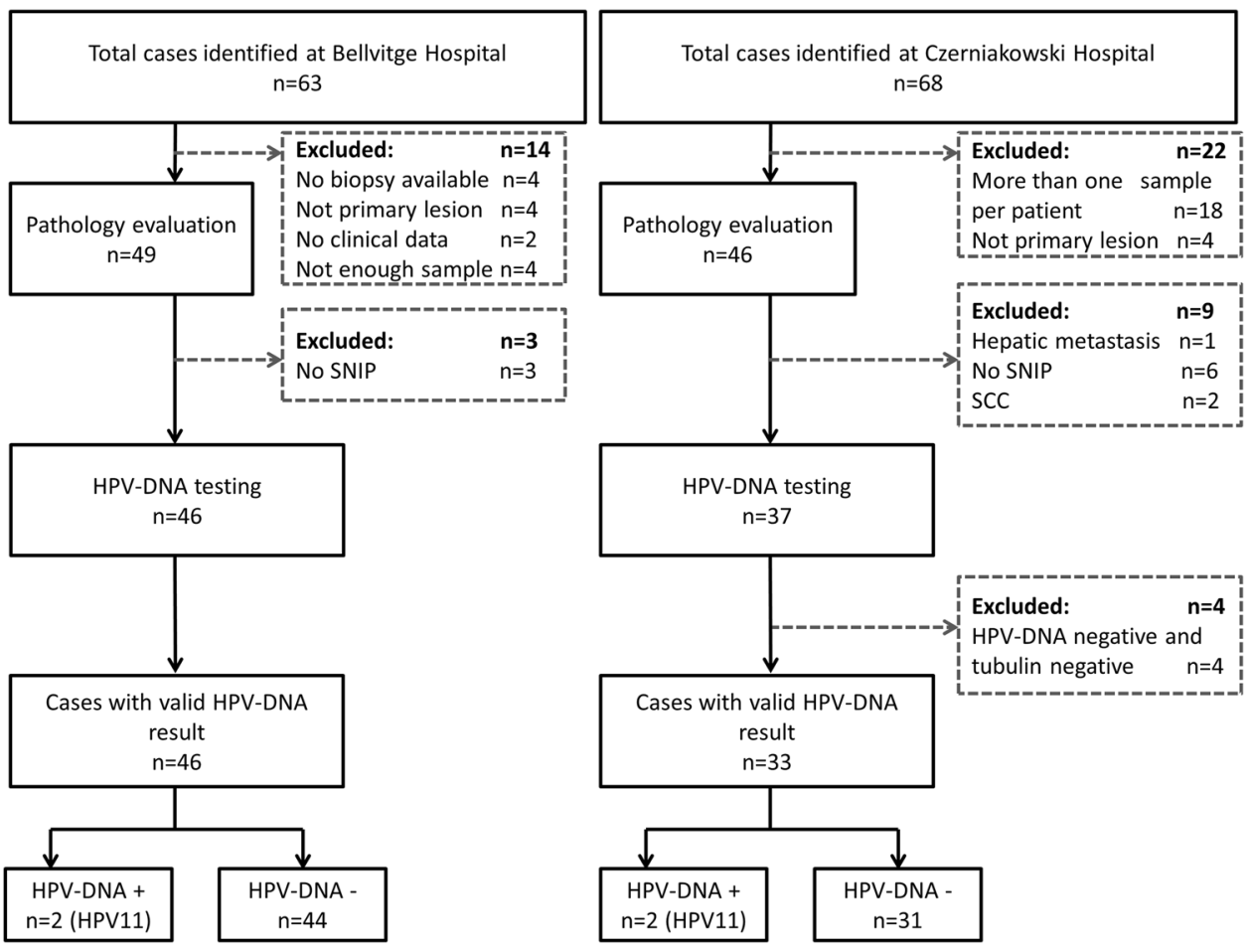

epithelium ( $\mathrm{p}=0.015)$ and more Spanish cases with squamous epithelium $(\mathrm{p}<0.001)$ and a papillary or exophytic lesion adjacent to SNIP ( $\mathrm{p}<0.001)$. The median time to follow-up was 76.63 months (range 0.23-174.3) for Spanish cases and 39.1 months (range 6.3-66.5) for Polish ones. Seventeen patients $(21.5 \%)$ had recurrence, of which 12 were from Spain (26.1\%) and 5 from Poland (15.2\%). The median time to recurrence was 14 months (range from 3 to 83 ). Only two cases $(2.5 \%)$, both belonging to the Spanish series, progressed to invasive cancer during the followup period. HPV-DNA was detected in two samples $(4.3 \%)$ in the Spanish series and two samples $(6.1 \%)$ in the Polish series. All of them were positive for HPV11 and negative for $\mathrm{p} 16^{\mathrm{INK} 4 \mathrm{a}}$ high expression. All HPV-DNA negative cases tested for $\mathrm{p} 16^{\mathrm{INK} 4 \mathrm{a}}$ (20 cases, representing $27 \%$ of all HPVDNA negative cases) were negative.

The presence of atypia adjacent to SNIP at diagnosis was the only statistically significant factor associated with recurrence with a crude hazard ratio (HR) of 18.83 (95\%CI, 1.71-207.65) (Table 2). The recurrence rate was higher with increased Krouse stage (T2 and T3) compared to T1, although this was not statistically significant. No significant differences in risk of recurrence were associated with smoking, alcohol intake, or HPV positivity. The four low riskHPV positive SNIPs were located in the nasal cavity (one of the septum, one of the vestibule, and two in the lateral wall. Among those, one in the lower turbinate and one in the middle turbinate and middle meatus). None of them presented with dysplasia. In contrast, most HPV-negative SNIPs were located at the lateral wall $(85 \%)$, the maxillary sinus $(15 \%)$ and the ethmoid sinus (20\%) with some affecting more than one location. The HPV-positive group comprised two males and two females with a mean age of 37.8 years old at the time of diagnosis. SNIPs recurred in $25 \%$ (1/4) of HPVpositive vs $22.2 \%(16 / 72)$ of HPV-negative lesions (crude $\mathrm{HR}=3.70,95 \% \mathrm{CI} 0.44-31.37$ ). Univariate analysis of the effects of tobacco use and Krouse stage on recurrence were stratified by centre, and statistically significant differences were not found.

\section{Discussion}

The etiologic and prognostic role of HPV in SNIP remains unclear, with previous studies reporting HPV detection rates ranging from 0 to $100 \%$ and contradictory results for the role of HPV infection in recurrence and malignant transformation [1, 3, 6, 10-12].The differences have not been explained by geographic region or HPV detection methods; however, to the best of our knowledge, no study has evaluated cases from different regions with the same sample processing and HPV detection protocols thus far. While published systematic reviews also pool findings from different geographic locations, our results are additive since systematic reviews and meta-analyses are not exempt from limitations [10, 18].

We evaluated the prevalence and prognostic role of HPV in two retrospective cohorts of primary SNIPs from Spain and Poland, as well as additional factors theorized to 
Table 1 Demographic and clinical characteristics of SNIP patients included in the study

\begin{tabular}{|c|c|c|c|c|}
\hline \multirow[t]{2}{*}{ Characteristics } & $\begin{array}{l}\text { Total } \\
\text { SNIPs } \\
(n=79)\end{array}$ & $\begin{array}{l}\text { Spanish } \\
\text { SNIPs } \\
(\mathrm{n}=46)\end{array}$ & $\begin{array}{l}\text { Polish } \\
\text { SNIPs } \\
(n=33)\end{array}$ & p-value ${ }^{a}$ \\
\hline & No. $(\%)^{\mathrm{a}}$ & No. $(\%)$ & No. $(\%)$ & \\
\hline Age at diagnosis & & & & 0.460 \\
\hline Mean (SD) & $56.2(16.1)$ & $55.0(15.0)$ & $57.8(17.7)$ & \\
\hline Range & $19-91$ & $19-85$ & $23-91$ & \\
\hline Gender & & & & 0.150 \\
\hline Male & $53(67.1)$ & $34(73.9)$ & $19(57.6)$ & \\
\hline Female & $26(32.9)$ & $12(26.1)$ & $14(42.4)$ & \\
\hline Period of diagnosis & & & & $<0.001$ \\
\hline 1995-2000 & $7(8.9)$ & $7(15.2)$ & $0(0.0)$ & \\
\hline 2001-2006 & $17(21.5)$ & $17(37.0)$ & $0(0.0)$ & \\
\hline 2007-2012 & $24(30.4)$ & $22(47.8)$ & $2(6.1)$ & \\
\hline 2013-2017 & $31(39.2)$ & $0(0.0)$ & $31(93.9)$ & \\
\hline Tobacco use & & & & 0.010 \\
\hline Never smoker & $33(41.8)$ & $13(28.3)$ & $20(60.6)$ & \\
\hline Ever smoker & $44(55.7)$ & $31(67.4)$ & $13(39.4)$ & \\
\hline Missing & $2(2.5)$ & $2(4.3)$ & $0(0.0)$ & \\
\hline Alcohol use & & & & 0.616 \\
\hline Never drinker & $56(70.9)$ & $33(71.7)$ & $23(69.7)$ & \\
\hline Ever drinker & $21(26.6)$ & $11(23.9)$ & $10(30.3)$ & \\
\hline Missing & $2(2.5)$ & $2(4.3)$ & $0(0.0)$ & \\
\hline Previous history of HPV-related pathology & & & & 1.000 \\
\hline Yes & $3(3.8)$ & $2(4.3)$ & $1(3.0)$ & \\
\hline No & $74(93.7)$ & $42(91.3)$ & $32(97.0)$ & \\
\hline Missing & $2(2.5)$ & $2(4.3)$ & $0(0.0)$ & \\
\hline Krouse classification & & & & $<0.001$ \\
\hline 1 & $33(41.8)$ & $28(60.9)$ & $5(15.2)$ & \\
\hline 2 & $23(29.1)$ & $11(23.9)$ & $12(36.4)$ & \\
\hline 3 & $16(20.3)$ & $3(6.5)$ & $13(39.4)$ & \\
\hline 4 & $4(5.1)$ & $1(2.2)$ & $3(9.1)$ & \\
\hline Missing & $3(3.8)$ & $3(6.5)$ & $0(0.0)$ & \\
\hline Transitionalepithelium & & & & 0.015 \\
\hline Absence & $19(24.1)$ & $16(34.8)$ & $3(9.1)$ & \\
\hline Presence & $60(75.9)$ & $30(65.2)$ & $30(90.9)$ & \\
\hline Squamousepithelium & & & & 0.001 \\
\hline Absence & $45(57.0)$ & $19(41.3)$ & $26(78.8)$ & \\
\hline Presence & $34(43.0)$ & $27(58.7)$ & $7(21.2)$ & \\
\hline Columnarepithelium & & & & 0.316 \\
\hline Absence & $23(29.1)$ & $11(23.9)$ & $12(36.4)$ & \\
\hline Presence & $56(70.9)$ & $35(76.1)$ & $21(63.6)$ & \\
\hline Hyper-parakeratosis & & & & 1 \\
\hline Absence & $76(96.2)$ & $44(95.7)$ & $32(97.0)$ & \\
\hline Presence & $3(3.8)$ & $2(4.3)$ & $1(3.0)$ & \\
\hline Papillar or exophytic lesion adjacent to SNIP & & & & $<0.001$ \\
\hline Absence & $61(77.2)$ & $44(95.7)$ & $17(51.5)$ & \\
\hline Presence & $18(22.8)$ & $2(4.3)$ & $16(48.5)$ & \\
\hline
\end{tabular}


Table 1 (continued)

\begin{tabular}{|c|c|c|c|c|}
\hline \multirow[t]{2}{*}{ Characteristics } & $\begin{array}{l}\text { Total } \\
\text { SNIPs } \\
(\mathrm{n}=79)\end{array}$ & $\begin{array}{l}\text { Spanish } \\
\text { SNIPs } \\
(\mathrm{n}=46)\end{array}$ & $\begin{array}{l}\text { Polish } \\
\text { SNIPs } \\
(\mathrm{n}=33)\end{array}$ & p-value ${ }^{a}$ \\
\hline & No. $(\%)^{\mathrm{a}}$ & No. (\%) & No. $(\%)$ & \\
\hline Polymorphonuclear neutrophil inflammatory intralesional infiltrate & & & & 1.000 \\
\hline Absence & $2(2.5)$ & $1(2.2)$ & $1(3.0)$ & \\
\hline Presence & $77(97.5)$ & $45(97.8)$ & $32(97.0)$ & \\
\hline Polymorphonuclear neutrophil inflammatory perilesional infiltrate & & & & 0.418 \\
\hline Absence & $1(1.3)$ & $0(0.0)$ & $1(3.0)$ & \\
\hline Presence & $78(98.7)$ & $46(100.0)$ & $32(97.0)$ & \\
\hline Recurrence & & & & 0.268 \\
\hline No & $59(74.7)$ & $31(67.4)$ & $28(84.8)$ & \\
\hline Yes & $17(21.5)$ & $12(26.1)$ & $5(15.2)$ & \\
\hline Missing & $3(3.8)$ & $3(6.5)$ & $0(0.0)$ & \\
\hline \multicolumn{5}{|l|}{ Months of follow up } \\
\hline Median & 52.83 & 76.63 & 39.1 & $0.003^{b}$ \\
\hline Range & $0.23-174.33$ & $0.23-174.33$ & $6.27-66.47$ & \\
\hline Months to recurrence & & & & $0.469^{\mathrm{b}}$ \\
\hline Median & 14 & 17 & 4 & \\
\hline Range & $3-83$ & $9-83$ & $3-36$ & \\
\hline Progression to invasive cancer & & & & 0.502 \\
\hline No & $74(93.7)$ & $41(89.1)$ & $33(100.0)$ & \\
\hline Yes & $2(2.5)$ & $2(4.3)$ & $0(0.0)$ & \\
\hline Missing & $3(3.8)$ & $3(6.5)$ & $0(0.0)$ & \\
\hline HPV positivity & & & & 1.000 \\
\hline No & $75(94.9)$ & $44(95.7)$ & $31(93.9)$ & \\
\hline Yes & $4(5.1)$ & $2(4.3)$ & $2(6.1)$ & \\
\hline
\end{tabular}

Statistically significant $p$ values given in bold

SNIP Sinonasal Inverted Papilloma, SD standard deviation

${ }^{a}$ Fischer exact test with the exception of age and months to recurrence. where t-student test has been used to compare the median values between populations

${ }^{b}$ qreg (quantilte regression): test for equality of medians, equivalent for t-test for medians

increase risk of recurrence. We tested SNIPs following a previously validated robust, standardized, international protocol designed to provide estimates of HPV-attributable fractions (HPV-AFs) in HPV-related cancers [14].

Our study is the first to evaluate SNIPs from two different geographic regions which have previously shown marked differences in HPV-AFs in oropharyngeal carcinoma (OPC) [14]. Our data demonstrates a low HPV-DNA detection $(5.1 \%)$ in primary SNIPs, similar to the $4.9 \%$ estimated in the oral cavity of healthy populations or the $4 \%$ and $7 \%$ estimated in inflammatory nasal polyps and normal sinonasal mucosa $[10,19]$. These findings suggest that HPV is not a main etiologic factor for SNIPs. Our HPV prevalence estimates are in accordance with previous studies with similar numbers of cases, although lower than others [7, 10, 11, 20, 21]. The only HPV subtype detected within a subset of
SNIPs was the low-risk HPV11 which is concordant with other's results $[3,7,20]$. No high-risk types were detected in our study.

The prevalence of HPV in recurring SNIPs and those with dysplasia or adjacent SCC is estimated to be higher than in SNIPs without dysplasia [3]. We included only primary lesions and identified one HPV-positive case from Spain with dysplasia adjacent to the SNIP. Three out of four HPV-positive patients in our series were ever smokers, although the association between the two variables was not statistically significant $(\mathrm{p}=0.631)$. The low HPV prevalence in our series prevented further exploration into factors associated with HPV positivity; however, we did note a trend for HPV positive cases to affect the nasal cavity rather than the sinus and to involve younger people. These observations are similar to those of other studies [22]. 
Table 2 Hazard ratios for recurrence in SNIP patients included in the study

\begin{tabular}{|c|c|c|c|}
\hline Characteristics & $\begin{array}{l}\text { Total SNIPs } \\
(\mathrm{n}=76) \\
\text { No. }(\%)\end{array}$ & $\begin{array}{l}\text { Recurrences } \\
\text { No. }(\%)\end{array}$ & $\begin{array}{l}\text { Crude HR } \\
(95 \% \mathrm{CI})\end{array}$ \\
\hline \multicolumn{4}{|l|}{ Hospital } \\
\hline Bellvitge (Spain) & $43(56.58)$ & $12(27.91)$ & Ref. \\
\hline Czerniakowski (Poland) & $33(43.42)$ & $5(15.15)$ & $0.81(0.27-2.39)$ \\
\hline \multicolumn{4}{|l|}{ Age at diagnosis ${ }^{\mathrm{b}}$} \\
\hline Mean (SD) & $56.34(16.40)$ & - & $1.01(0.98-1.04)$ \\
\hline \multicolumn{4}{|l|}{ Gender } \\
\hline Male & $51(67.11)$ & $14(27.45)$ & Ref. \\
\hline Female & $25(32.89)$ & $3(12.00)$ & $0.45(0.13-1.58)$ \\
\hline \multicolumn{4}{|l|}{ Year of diagnosis } \\
\hline Range & $1995-2017$ & - & $0.96(0.86-1.08)$ \\
\hline \multicolumn{4}{|l|}{ Tobacco use } \\
\hline Never smoker & $32(42.11)$ & $7(21.88)$ & Ref. \\
\hline Ever smoker & $44(57.89)$ & $10(22.73)$ & $0.97(0.35-2.64)$ \\
\hline \multicolumn{4}{|l|}{ Alcohol use } \\
\hline Never drinker & $55(72.37)$ & $12(21.82)$ & Ref. \\
\hline Ever drinker & $21(27.63)$ & $5(23.81)$ & $1.32(0.46-3.78)$ \\
\hline \multicolumn{4}{|c|}{ Previous history of HPV-related pathology } \\
\hline No & $73(96.05)$ & $16(21.92)$ & Ref. \\
\hline Yes & $3(3.95)$ & $1(33.33)$ & $0.91(0.12-7.06)$ \\
\hline \multicolumn{4}{|l|}{ Krouse classification } \\
\hline 1 & $33(43.42)$ & $8(24.24)$ & Ref. \\
\hline 2 & $23(30.26)$ & $6(26.09)$ & $1.70(0.54-5.29)$ \\
\hline 3 & $16(21.05)$ & $3(18.75)$ & $1.27(0.28-5.75)$ \\
\hline 4 & $4(5.26)$ & $0(0.00)$ & - \\
\hline \multicolumn{4}{|l|}{ Transitional epithelium } \\
\hline Absence & $18(23.68)$ & $2(11.11)$ & Ref. \\
\hline Presence & $58(76.32)$ & 15 (25.86) & $3.01(0.67-13.56)$ \\
\hline \multicolumn{4}{|l|}{ Squamous epithelium } \\
\hline Absence & $43(56.58)$ & $11(25.58)$ & Ref. \\
\hline Presence & $33(43.42)$ & $6(18.18)$ & $0.49(0.17-1.43)$ \\
\hline \multicolumn{4}{|l|}{ Columnar epithelium } \\
\hline Absence & 22 (28.95) & $3(13.64)$ & Ref. \\
\hline Presence & 54 (71.05) & $14(25.93)$ & $1.90(0.54-6.69)$ \\
\hline \multicolumn{4}{|c|}{ Papillar or exophytic lesion adyacent to SNIP } \\
\hline Absence & $58(76.32)$ & $14(24.14)$ & Ref. \\
\hline Presence & $18(23.68)$ & $3(16.67)$ & $0.85(0.20-3.55)$ \\
\hline \multicolumn{4}{|c|}{ Polymorphonuclear neutrophil inflammatory intralesional infiltrate (present in 74 cases) } \\
\hline Low & 67 (90.54) & $16(23.88)$ & Ref. \\
\hline Moderate & $7(9.46)$ & $1(14.29)$ & $0.52(0.07-3.96)$ \\
\hline \multicolumn{4}{|c|}{ Polymorphonuclear neutrophil inflammatory perilesional infiltrate (present in 75 cases) } \\
\hline Low & $56(74.67)$ & $12(21.43)$ & Ref. \\
\hline Moderate & $18(24.00)$ & $5(27.78)$ & $2.59(0.79-8.52)$ \\
\hline Severe & $1(1.33)$ & $0(0.00)$ & - \\
\hline \multicolumn{4}{|c|}{ Dysplasia at diagnosis adyacent to SNIP } \\
\hline Absence & 75 (98.68) & $16(21.33)$ & Ref. \\
\hline Presence & $1(1.32)$ & $1(100.00)$ & $18.83(1.71-207.65)$ \\
\hline \multicolumn{4}{|c|}{ Progression to cancer during follow-up } \\
\hline No & $74(97.37)$ & $16(21.62)$ & Ref. \\
\hline Yes & $2(2.63)$ & $1(50.00)$ & $1.69(0.22-13.24)$ \\
\hline
\end{tabular}


Table 2 (continued)

\begin{tabular}{llll}
\hline Characteristics & $\begin{array}{l}\text { Total SNIPs } \\
(\mathrm{n}=76) \\
\text { No. }(\%)\end{array}$ & $\begin{array}{l}\text { Recurrences } \\
\text { No. }(\%)\end{array}$ & $\begin{array}{l}\text { Crude HR } \\
(95 \% \mathrm{CI})\end{array}$ \\
\hline HPV positivity & & $16(22.22)$ & Ref. \\
No & $72(94.74)$ & $1(25.00)$ & $3.70(0.44-31.37)$ \\
Yes & $4(5.26)$ & &
\end{tabular}

Statistically significant confidence interval given in bold

SNIP Sinonasal Inverted Papilloma

${ }^{\text {a }}$ Three out of 79 cases did not have information regarding recurrence

${ }^{\mathrm{b}}$ First row shows mean age of the sample with its standard deviation

We identified some regional differences between the two studied, with Polish cases diagnosed at more recent periods (due to case selection), more advanced Krouse stages, and a lower proportion of ever-smokers. Histopathologic features also varied between Polish and Spanish cases, namely, the type of epithelium present. Despite the differences, due the low case number, we decided to combine groups to evaluate factors associated with recurrence and address the differences with the use of the strata function in Stata. When accounting for such differences by univariate Cox models for effects of tobacco use and Krouse stage on recurrence by centre, we did not find statistically significant differences between groups. Although showing marked differences in HPV-AF in OPC for SNIPs, both series showed similar HPV prevalence, confirming that the variability in HPV detection rates in SNIPs is not explained by their different geographic origins as was already hypothesized in a previous meta-analysis [10]

The presence of dysplasia adjacent to the SNIP at diagnosis was the only factor associated with recurrence (HR: 18.83, 95\% CI 1.71-207.65), as shown in previous studies, although this was only identified in one case in our series [23]. A HR of 3.70 (95\% CI 0.44-31.37) for recurrence in HPV-positive cases seen but not statistically significant. Neither tobacco smoking or Krouse stage were associated with recurrence in our series but both are implicated in others [3, $7,8]$. The low number of cases evaluated in this study could explain the discrepancies with the literature.

Only two cases (2.6\%) progressed to invasive cancer during follow-up and neither were HPV-positive. Therefore, the prognostic value of HPV positivity or other factors for malignant transformation was not further evaluated in this study. Other risk factors suspected to be involved in malignant transformation of SNIPs include HPV infection, tobacco smoking, and occupational exposures [24]. In contrast, EGFR mutations have been observed to protect against malignant transformation in SNIPs [21, 24]. A recent study showed that EGFR mutations and HPV infection represent essential, alternative oncogenic mechanisms in SNIP and SNIP-associated sinonasal SCC [21]. The study observed that SNIP progression was significantly associated with the presence of HPV infection and the absence of an EGFR mutation.

Previous studies that suggest HPV infection may play a role as a co-factor in the development of carcinoma exSNIP neglected to use biomarkers of biologic activity of HPV such as the presence of E6/E7 mRNA transcripts or p16 ${ }^{\mathrm{INK} 4 \mathrm{a}}$ expression. Several studies that evaluated E6/E7 mRNA transcripts or $\mathrm{p} 16^{\mathrm{INK} 4 \mathrm{a}}$ expression in SNIPs did not find HPV to be an etiologic driver of SNIP development or progression to SCC $[25,26]$. We did not observe p16 ${ }^{\text {INK4a }}$ expression in any HPV-DNA positive samples as expected, since all of them were positive for low risk genotypes. While not all HPV-DNA negative samples were tested for p $16^{\text {INK4a }}$, the 20 tested were negative for expression. We did not evaluate further biomarkers of biologic activity of HPV on HPV-DNA positive samples such as E6/E7 mRNA positivity or use techniques like laser capture microdissection (LCM). LCM, when combined with highly sensitive PCR, allows assignment of a particular HPV genotype to an area of normal or abnormal epithelium [27].

The major limitation of the study was its relatively small sample size, which precluded the evaluation of factors associated with HPV-positivity, recurrence, and malignant transformation. Despite the small sample size and given the fact that SNIP is a relatively rare entity, few studies have reported results for series with similar numbers of primary SNIP cases, consecutively diagnosed in two decades, like ours. Not all HPV-DNA negative cases were evaluated for $\mathrm{p} 16^{\mathrm{INK} 4 \mathrm{a}}$ expression and no evaluation of further biomarkers such as E6/E7 mRNA or EGFR was performed. Furthermore, we did not evaluate the prognostic value of the treatment received. However, a previous study did not find differences in recurrence by different types of interventions [9]. 


\section{Conclusion}

The low prevalence of HPV-DNA found in SNIPs from two different countries suggests that HPV is not a main etiologic factor for SNIP. The lack of an association between HPV and other evaluated factors with recurrence may suggest the involvement of other mechanisms. Further research with larger numbers of patients and additional biomarkers is warranted to unequivocally assess the etiology and prognosis of SNIP.

Funding We thank CERCA Program/Generalitat de Catalunya for institutional support. This study has been funded by the Instituto de Salud Carlos III (ie, the Spanish government), the European Regional Development Fund-A Way to Build Europe, through the projects CIBERESP CB06/02/0073 and CIBERONC, CB16/12/0040, from the Agència de Gestió d'Ajuts Universitaris i de Recerca (2017SGR1085) and from the Department of Health of the Generalitat de Catalunya (PERIS-2016-2020, SLT002/16/00404) (personal grants to MM and JFG, respectively).

\section{Compliance with Ethical Standards}

Conflict of interest Cancer Epidemiology Research Program (LA MM JF BQ OC MP) has received sponsorship for grants from Merck and co, Roche, Reig-jofre, IDT, Hologic, GlaxoSmithKline and Seegene. The rest of authors have declared no conflicts of interest.

\section{References}

1. Bishop JA. OSPs and ESPs and ISPs, Oh My! An Update on Sinonasal (Schneiderian) Papillomas. Head Neck Pathol. 2017;11(3):269-77.

2. Hyams VJ. Papillomas of the nasal cavity and paranasal sinuses. A clinicopathological study of 315 cases. Ann Otol Rhinol Laryngol. 1971;80(2):192-206

3. Lawson W, Schlecht NF, Brandwein-Gensler M. The role of the human papillomavirus in the pathogenesis of Schneiderian Inverted papillomas: an analytic overview of the evidence. Head Neck Pathol. 2008;2:49-59.

4. Sung-Lyong Hong MD, Bae-Hyun Kim MD, Jung-Hoon Lee MD, Kyu-Sup Cho MD, Hwan-Jung Roh MD. Smoking and malignancy in sinonasal inverted papilloma. Laryngoscope. 2013;123:1087-91.

5. Re M, Gioacchini FM, Bajraktari A, Tomasetti M, Kaleci S, Rubini C, Bertini A, Magliulo G, Pasquini E. Malignant transformation of sinonasal inverted papilloma and related genetic alterations: a systematic review. Eur Arch Otorhinolaryngol. 2017;274(8):2991-3000.

6. Beck JC, McClatchey KD, Lesperance MM, Esclamado RM, Carey TE, Bradford CR. Presence of human papillomavirus predicts recurrence of inverted papilloma. Otolaryngol Head Neck Surg. 1995;113:49-55.

7. Roh HJ, Mun SJ, Cho KS, Hong SL. Smoking, not human papilloma virus infection, is a risk factor for recurrence of sinonasal inverted papilloma. Am J Rhinol Allergy. 2016;30(2):79-82.

8. Lisan Q, Moya-Plana A, Bonfils P. Association of Krouse classification for sinonasal inverted papilloma with recurrence: a systematic review and meta-analysis. JAMA Otolaryngol Head Neck Surg. 2017;143(11):1104-10.

9. Attlmayr B, Derbyshire SG, Kasbekar AV, Swift AC. Management of inverted papilloma: review. J Laryngol Otol. 2017;131(4):284-9.

10. Syrjanen K, Syrjanen S. Detection of human papillomavirus in sinonasal papillomas: systematic review and meta-analysis. Laryngoscope. 2013;123:181-92.

11. Justice JM, Davis KM, Saenz DA, Lanza DC. Evidence that human papillomavirus causes inverted papilloma is sparse. Int Forum Allergy Rhinol. 2014;4:995-1001.

12. Zhao RW, Guo ZQ, Zhang RX. Human papillomavirus infection and the malignant transformation of sinonasal inverted papilloma: A meta-analysis. J Clin Virol. 2016;79:36-433.

13. Krouse JH. Development of a staging system for inverted papilloma. Laryngoscope. 2000;110:965-8.

14. Castellsagué X, Alemany L, Quer M, et al. HPV involvement in head and neck cancers: comprehensive assessment of biomarkers in 3680 patients. J Natl Cancer Inst. 2016;108(6):djv403.

15. Westra WH. Detection of human papillomavirus (HPV) in clinical samples: Evolving methods and strategies for the accurate determination of HPV status of head and neck carcinomas. Oral Oncol. 2014;50(9):771-9.

16. Halec G, Holzinger D, Schmitt M, Flechtenmacher C, Dyckhoff G, Lloveras B, et al. Biological evidence for a causal role of HPV16 in a small fraction of laryngeal squamous cell carcinoma. Br J Cancer. 2013;109(1):172-83.

17. Gomez-Armayones S, Chimenos-Küstner E, Marí A, Tous S, Penin R, Clavero O, Quiros B, Pavón MA, Taberna M, Alemany L, Servitje O, Mena M. Human papillomavirus in premalignant oral lesions: no evidence of association in a Spanish cohort. PLoS ONE. 2019;14(1):e0210070.

18. Greenland S. Can meta-analyses be salvaged? Am J Epidemiol. 1994;140:783-7.

19. Mena M, Taberna M, Monfil L, et al. Might oral HPV in healthy individuals explain differences in HPV-attributable fractions in oropharyngeal cancer? A systematic review and meta-analysis. J Infect Dis. 2018. https://doi.org/10.1093/infdis/jiy715.

20. Scheel A, Lin GC, McHugh JB, et al. Human papillomavirus infection and biomarkers in sinonasal inverted papillomas: clinical significance and molecular mechanisms. Int Forum Allergy Rhinol. 2015;5(8):701-7.

21. Udager AM, McHugh JB, Goudsmit CM, et al. Human papillomavirus (HPV) and somatic EGFR mutations are essential, mutually exclusive oncogenic mechanisms for inverted sinonasal papillomas and associated sinonasal squamous cell carcinomas. Ann Oncol. 2018;29(2):466-71.

22. Elliot A, Näsman A, Westman M, et al. Human papillomavirus and infiltration of CD8- and Foxp3-positive immune cells in sinonasal inverted papillomas. Acta Oto Laryngol. 2019;139(11):1651-2251.

23. Safadi A, Yafit D, Abu-Ghanem S, et al. The clinical behavior of sinonasal inverted papilloma with cellular dysplasia: case series and review of the literature. Eur Arch Otorhinolaryngol. 2017;274(9):3375-82.

24. Sahnane N, Ottini G, Turri-Zanoni M, et al. Comprehensive analysis of HPV infection, EGFR exon 20 mutations and LINE1 hypomethylation as risk factors for malignant transformation of sinonasal-inverted papilloma to squamous cell carcinoma. Int J Cancer. 2019;144(6):1313-20.

25. Rooper LM, Bishop JA, Westra WH. Transcriptionally active high-risk human papillomavirus is not a common etiologic agent in the malignant transformation of Inverted schneiderian papillomas. Head NeckPathol. 2017;11(3):346-53. 
26. Mohajeri S, Lai C, Purgina B, et al. Human papillomavirus: An unlikely etiologic factor in sinonasal inverted papilloma. Laryngoscope. 2018;128(11):2443-7.

27. Guimerà N, Lloveras $\mathrm{B}$, Alemany L, et al. Laser capture microdissection shows HPV11 as both a causal and a coincidental infection in cervical cancer specimens with multiple HPV types. Histopathology. 2013;63(2):287-92.
Publisher's Note Springer Nature remains neutral with regard to jurisdictional claims in published maps and institutional affiliations. 